\title{
PROCAM y Framingham por categorías: ¿miden igual riesgo?
}

\author{
A. Álvarez Cosmea, V. López Fernández, M. A. Prieto díaz*, L. Díaz Gonzá- \\ lez, P. Herrero Puente**, J. Vázquez Álvarez**, S. Fidalgo González* \\ Médicos de M ediana Familiar y Comunitaria. \\ C.S. Ventanielles. *C.S. Vallobin- Concinos. **Servicio de U rgencias. \\ Hospital Central de Asturias. Oviedo. Principado de Asturias
}

\section{RESUMEN}

Objetivo: comparar dos métodos de cálculo de riesgo cardiovascular, PROCAM y Framingham por categorías (eventos duros), en varones entre 45-65 años.

Material y métodos: estudio descriptivo transver sal. Ámbito del estudio: Atención Primaria. La po blación a estudio fueron todos los varones de 4565 años de cuatro cupos de Medicina General de tres Centros de Salud, sin enfermedad cardiovascu lar establecida $(n=402)$. Las variables fueron: edad, diabetes mellitus, tabaquismo, antecedentes familiares de enfermedad coronaria precoz, pre sión arterial sistólica y diastólica, colesterol total, LDL-colesterol, HDL-colesterol, triglicéridos. El periodo de estudio fue del 1 de enero a 31 de di ciembre de 1999. Para comparar ambos métodos calculamos el coeficiente de correlación de Pear son, coeficiente de correlación intraclase (CCI) y se hizo la representación gráfica usando el modelo de Bland y Altman.

Resultados: la media de riesgo al utilizar PROCAM es de 8,56 \pm 9,3\%, frente a 10,85 $\pm 6,8 \%$ del Fra mingham por categorías; el coeficiente de correla ción de Pearson es de 0,859 ( $p<0,001)$, el CCI fue de $0,778$ ( $p<0,001)$. La diferencia media PROCAMFramingham ha sido de $-2,2 \% \pm 4,9 p<0,001$.

Conclusiones: a pesar de tener coeficientes de correlación superiores a 0,75 y que la media de las diferencias es de sólo 2,2\%, existe una gran variabilidad en las diferencias individuales a me. dida que el riesgo aumenta. Para riesgos bajos,
PROCAM and Framinghan using risk factors ca tegories: does it mesure the same risk?

\begin{abstract}
Objective: to compare two methods to calculate cardiovascular risk, PROCAM and Framingham using risk factors categories (hard events), in men between 45 and 65 years.
\end{abstract}

Material and methods: we present a descriptive crossover study, made between January and De cember of 1999. The study was made in Primary Heatlh Care. We have examined all of men between 45 and 65 years, which belong to four family doc tors in three health centers, without cardiovascular disease ( $n=402)$. For every man, it was analysed: age, diabetes mellitus, smoke, family history of pre mature coronary heart disease, systolic blood pres sure, diastolic blood pressure, total cholesterol, LDL-cholesterol, HDL-cholesterol, tryglicerides. To compare both methods we used Pearson corre lation coefficient, intraclass correlation coefficient (ICC), and the graphic representation was made with the Bland and Altman model.

Results: average cardiovascular risk was 8.56 $\pm 9.3 \%$ with PROCAM method versus $10.85 \pm$ $6.8 \%$ with Framingham using risk factors catego ries. Pearson correlation coefficient was 0.859 ( $p<0.001)$, ICC was 0.778 ( $p<0.001)$. Average difference between PROCAM and Framingham using risk factors categories was $-2.2 \% \pm 4.9 p<$ 0.001 .

Conclusions: even the correlation coefficients are higher than 0.75, and the average difference are $2.2 \%$, there are a great variability in the individuals

Aceptación: 17-01-02 
PROCAM infravalora el riesgo, mientras que lo supravalora para riesgos altos, comparado con Framingham por categorías.

Palabras clave: Prevención. Riesgo coronario. Cardiopatía isquémica. differences with the increase risk. Compared with Framingham using risk factors categories, for a low risk, PROCAM underestimates this risk, while, for a high risk PROCAM overvalues the risk.

Key words: Prevention. Coronary risk assess ment. Ischaemic heart disease.

\section{INTRODUCCIÓN}

En los últimos años, las distintas Sociedades Científicas han puesto mucho énfasis en que para el tratamiento con fármacos de los distintos factores de riesgo, sobre todo la hipercolesterolemia, se debe realizar una aproximación al riesgo cardiovascular global de ese individuo, y para ello existen varios métodos o escalas de riesgo cardiovascular; entre las más conocidas podemos destacar las tablas de riesgo Framingham (clásica, categorías, D’Agostino) $)^{1-5}$. Sociedades Europeas ${ }^{6}$, Británicas ${ }^{7,8}$, Nueva Zelanda ${ }^{9}$, Sheffiel ${ }^{10}$, Sociedad Internacional de Arteriosclerosis ${ }^{11}$, etc., casi todas ellas, se basan en los resultados del estudio de Framingham, con algunas excepciones, entre las que destaca la escala de riesgo del Prospective Cardiovascular Münster Study (PROCAM) ${ }^{12}$, estudio realizado en Münster, Alemania.

El objetivo fundamental de nuestro estudio ha sido comparar si dos métodos de cálculo de riesgo cardiovascular, PROCAM (Alemania) y Framingham por Categorías (EE.UU.), miden igual riesgo en una población de varones entre 45 y 65 años.

\section{MATERIAL Y MÉTODO}

Se realizó un estudio transversal en el ámbito de Atención Primaria, siendo la población a estudio todos los varones entre 45 y 65 años de edad, de cuatro cupos de medicina general de tres centros de salud de la comunidad autónoma del Principado de Asturias. Para confeccionar el listado de sujetos, se utilizó la base de datos de tarjeta sanitaria individual de los referidos cupos, en total 600 varones. Consideramos criterios de exclusión: enfermos terminales, inmovilizados, alcohólicos, trastornos mentales severos y los pacientes que no residían en la zona, no se localizaron o no querían participar en el estudio tras haber sido informados.

La captación de los sujetos se realizó a partir de la consulta a demanda y en el caso de los que no acudían, se concertaba una consulta telefónica para realizar el estudio completo; el periodo de estudio fue de enero a diciembre de 1999.
Se realizó una hoja específica de recogida de datos con las siguientes variables: edad, antecedentes familiares en primer grado de enfermedad coronaria precoz o muerte súbita en varones menores de 55 años y en mujeres menores de 65 años, antecedentes personales documentados con informe médico de patología cardiovascular (cardiopatía isquémica, accidente cerebrovascular, insuficiencia cardiaca e isquemia crónica de miembros inferiores), presión arterial sistólica y diastólica (se descartaba la primera toma en consulta y se realizaron otras dos tomas, siendo el resultado final la media de estas dos últimas), diabetes (se consideraron como diabéticos aquellos pacientes ya diagnosticados o con tratamiento antidiabético; para el nuevo diagnóstico se utilizaron los criterios de la OMS), hábito tabáquico (se consideraron fumadores aquéllos que han fumado al menos 1 cigarrillo en el último mes), perfil lipídico completo (colesterol total, triglicéridos, HDL-colesterol, LDL-colesterol).

Para el cálculo de riesgo cardiovascular se utilizaron 2 escalas de riesgo: a) PROCAM: las variables que utiliza son edad, LDL-colesterol, HDLcolesterol, triglicéridos, tabaco, diabetes y antecedente familiar de infarto agudo de miocardio (IAM) menor de 60 años; estima el riesgo en un periodo de 10 años de IAM fatal y no fatal, basado en un estudio de 4.501 varones entre 40-65 años realizado en Münster (Alemania); para el cálculo utilizamos la página web de la International Task Force for Prevention of Coronary Heart Disease ${ }^{13}$ $\mathrm{y}$ es el método recomendado por la Sociedad Internacional de Arteriosclerosis ${ }^{11}$; y b) tablas de riesgo de Framingham por categorías: adaptación de la tabla de riesgo de Framingham original al V Joint National Committee ${ }^{14}$ y al National Colesterol Edu cation Program $^{15}$, teniendo en cuenta las variables de edad, colesterol total, HDL-colesterol, presión arterial sistólica y diastólica, tabaco y diabetes; se suman los puntos de las distintas variables y obtenemos el riesgo a 10 años de presentar IAM no fatal o muerte coronaria; las diferencias entre ambos métodos quedan reflejados en la tabla I. La población diana fueron todos los varones sin enfermedad cardiovascular previa.

Análisis estadístico: se utilizó para el análisis de los datos el programa SPSS 10,0; a cada paciente 
Tabla I

DIFERENCIAS RESPECTO A VARIABLES ENTRE PROCAM Y FRAMINGHAM POR CATEGORIAS

\begin{tabular}{lcc}
\hline & PROCAM & Framingham por categorías \\
\hline Edad & $40-65$ años & 30-74 años \\
Sexo & Hombres & Hombres y mujeres \\
Colesterol total & No & Sí* \\
LDL-colesterol & Sí & Sí* \\
HDL-colesterol & Sí & Sí \\
Triglicéridos & Sí & No \\
Presión A. Sistólica & Sí & Sí \\
Presión A. Diastólica & No & Sí \\
Diabetes & Sí/ No & Sí/ No \\
Tabaco & Sí/ No & Sí/ No \\
Antecedentes Familiares & IAM <60 años $<$ No \\
Riesgo Coronario & IAM fatal y no fatal & IAM y muerte coronaria** \\
\hline
\end{tabular}

* Podemos utilizar indistintamente el colesterol total o LDL-colesterol

** Se puede calcular el riesgo de enfermedad coronaria total (angina, IAM o muerte coronaria) o eventos duros (IAM y muerte coronaria).

se estimó el riesgo de presentar un evento por ambos métodos (PROCAM y Framingham por categorías), expresado en porcentaje a los 10 años. Para comparar ambos métodos de riesgo se calculó el coeficiente de correlación de Pearson, el coeficiente de correlación Intraclase (CCI) y la representación gráfica de Bland y Altman. Para valorar si las diferencias tenían repercusión clínica se calificó el riesgo obtenido en dos categorías: alto riesgo (porcentaje a 10 años igual o superior al 20\%) y moderado-bajo riesgo (menor del 20\%); para comparar los resultados obtenidos por los dos métodos, se utilizó el coeficiente de Kappa.

\section{RESULTADOS}

De los 600 varones del listado de tarjetas sanitarias fueron excluidos 113 pacientes (19\%), siendo las causas: 44 no aceptaron participar, 34 no se captaron, 18 por vivir fuera de zona, 10 neoplasias de mal pronóstico, 5 por consumo excesivo de alcohol y 2 estaban muertos; de los 487 pacientes restantes, 49 tenían ya una patología cardiovascular manifiesta, por lo que quedaron 438 para el cálculo del riesgo coronario; si tenemos en cuenta que en 36 casos, el laboratorio o bien no realizó el HDLcolesterol o los triglicéridos fueron superiores a 400 y por tanto no pudimos calcular el LDL-colesterol, obtenemos una población diana de 402 pacientes.

Encontramos un 10,7\% de diabéticos, el $47 \%$ eran fumadores y el resto de las características generales queda reflejado en la tabla II; el coeficiente

\begin{tabular}{|c|c|}
\hline $\begin{array}{l}\text { CARACTERÍSTICAS GENERALES D } \\
\text { DE 45-65 AÑOS }\end{array}$ & LOS VARONES \\
\hline Edad (años) & $54,3 \pm 6,62$ \\
\hline Colesterol total (mg/ dl) & $229,5 \pm 40,6$ \\
\hline LDL- colesterol (mg/ dl) & $153,4 \pm 37$ \\
\hline HDL-colesterol (mg/ dl) & $49,5 \pm 12,4$ \\
\hline Triglicéridos (mg/ dl) & $146,2 \pm 89$ \\
\hline Presión Arterial Sistólica (mmHg) & $128,6 \pm 15,6$ \\
\hline Presión Arterial Diastólica (mmHg) & $78,11 \pm 9,45$ \\
\hline PROCAM (\% a los 10 años) & $8,56 \pm 9,3$ \\
\hline $\begin{array}{l}\text { Framingham categorías } \\
\text { ( } \% \text { a los } 10 \text { años) }\end{array}$ & $10,85 \pm 6,8$ \\
\hline
\end{tabular}

de correlación de Pearson entre los dos métodos de cálculo de riesgo es de $0,859(\mathrm{r}=0,859, \mathrm{p}<0,001)$ (Fig. 1) y el coeficiente de correlación Intraclase de $0,778$ ( $\mathrm{p}<0,001)$; la diferencia media de los riesgos (PROCAM- Framingham) es de $-2,2 \% \pm 4,6$ DT $(\mathrm{p}<0,001)$ y la representación gráfica de la diferencia entre ambos contra su media de riesgo (Método de Bland y Altman) queda reflejado en la figura 2.

Si definimos alto riesgo como la probabilidad de ocurrir un evento cardiovascular (IAM fatal y no fatal) $\geq 20 \%$ a los 10 años, al aplicar PROCAM se consideraría como tal a 35 pacientes $(9 \%)$, mientras que si utilizamos Framingham por Categorías 


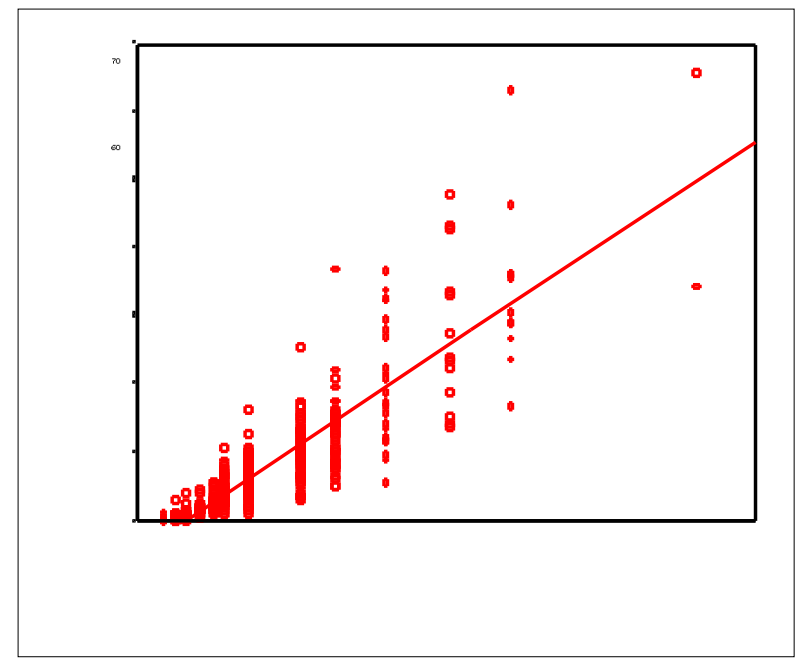

Figura 1

Relación entre PROCAM y Framingham por categorías

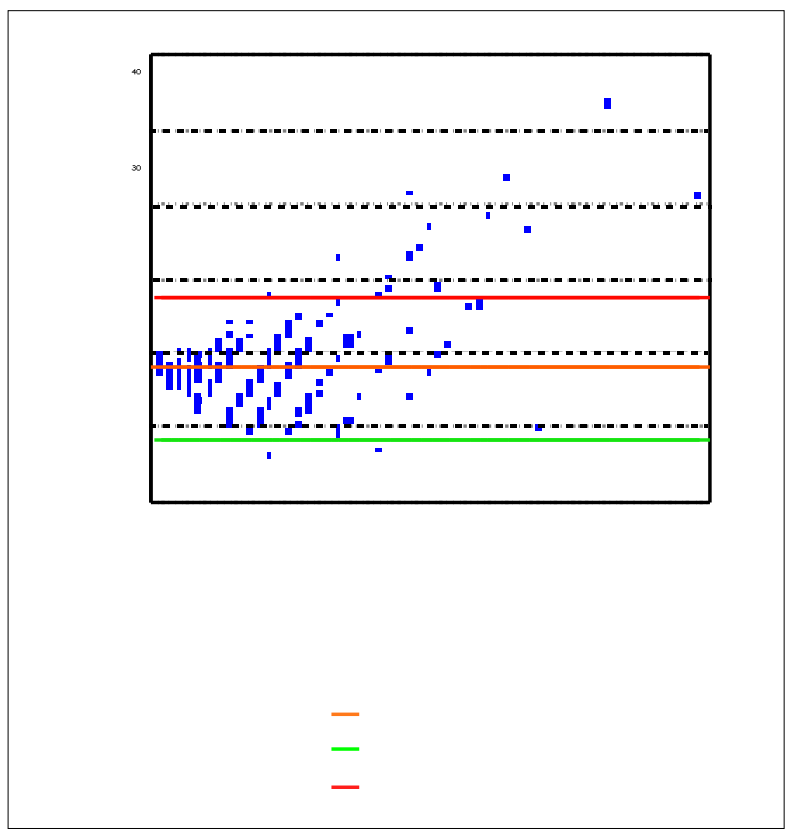

Figura 2

Gráfico de Bland y Almant

clasificaríamos como alto riesgo a 49 (12\%), con un Índice Kappa de 0,71 (Tabla III).

\section{DISCUSIÓN}

Casi todas la escalas de riesgo utilizadas en la práctica clínica, derivan del estudio de Framingham y aunque parecen ser válidas para otras poblaciones, como el Norte de Europa ${ }^{16}$, parece que pueden supraestimar el riesgo cardiovascular en

\section{Tabla III}

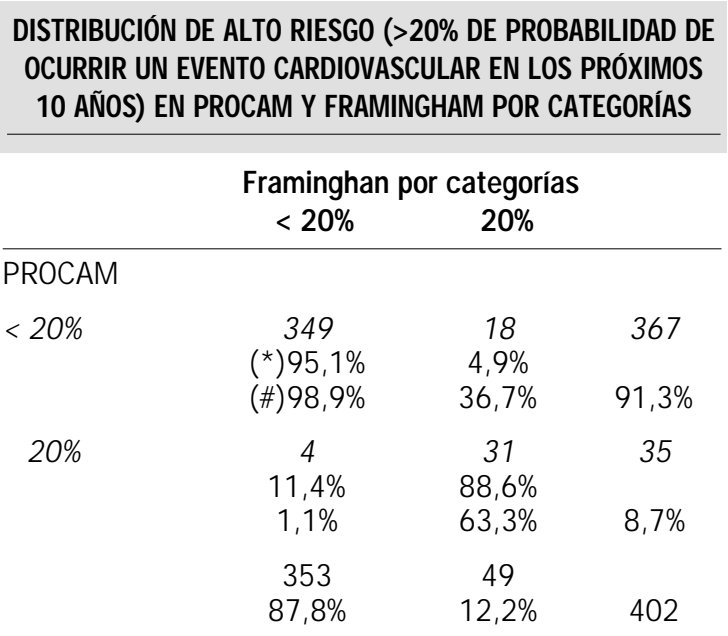

(*) Porcentaje en fila. (\#) Porcentaje en columna. Coeficiente kapa 0,71; error estándar de kappa 0,049 ( $p<0,000001)$.

poblaciones mediterráneas ${ }^{17,18} \mathrm{e}$ incluso en diversos grupos étnicos de América ${ }^{19}$; la escala de riesgo PROCAM está realizada en Europa (Münster, Alemania) y por lo tanto pretendimos comparar si miden igual riesgo en nuestra población de estudio.

La media de riesgo de PROCAM fue inferior a la estimada por Framingham por categorías $(8,56 \pm$ 9,3 frente a $10,85 \pm 6,8$ ), con una diferencia de $2,2 \pm 4,9$; en otros estudios realizados en población de mayor riesgo ${ }^{16}$, la diferencia no ha sido estadísticamente diferente de $0(\mathrm{p}>0,05)$.

Para la cuantificación de la concordancia de ambos métodos, utilizamos la $\mathrm{r}$ de Pearson siendo ésta de 0,859 , que según la interpretación de dicho coeficiente, podríamos decir que la correlación es buena o excelente; a pesar de ello, algunos autores consideran que no es el mejor índice para expresar concordancia, ya que dos medidas pueden estar relacionadas, pero no dar nunca el mismo resultado ${ }^{20}$; como medida de fiabilidad, el coeficiente de correlación intraclase (CCI) es mejor que la $\mathrm{r}$ de Pearson, siendo éste en nuestro caso de 0,778 ( $\mathrm{p}<$ $0,001)$ que, según la clasificación de Fermanian ${ }^{21}$ se considera buena.

El método de Bland y Altman ${ }^{22,23}$ ha alcanzado una gran difusión para analizar la concordancia entre dos métodos, consistiendo en representar gráficamente la diferencia entre dos observaciones (diferencia de riesgos) contra su media (media de riesgo), y nos informa explícitamente de las discrepancias entre cada par de observaciones; en nuestra población (Fig. 2), podemos apreciar que por debajo del $20 \%$ de media de riesgo, los puntos están más aglutinados y casi todos ellos por debajo de 0 , mientras que por encima del $20 \%$, existe una mayor dispersión de observaciones y casi todas ellas 
por encima de 0; dado que en el eje de las ordenadas tenemos PROCAM - Framingham, podemos decir que para riesgos menores del 20\%, PROCAM infravalora el riesgo, mientras que para riesgos altos lo supravalora comparado con Framingham por categorías; en poblaciones de mayor riesgo, también se dan los mismos resultados ${ }^{16}$.

Respecto a la clasificación de alto riesgo $(\geq 20 \%)$, presenta un índice Kappa de 0,71, que expresa un grado de concordancia buena ${ }^{23}$; en la tabla III, podemos apreciar que de los 35 pacientes que PROCAM considera de alto riesgo, solamente 4 (11\%) no se considerarían como tales si aplicamos Framingham; sin embargo de los 49 que Framingham etiqueta de alto riesgo, un $37 \%$ no lo serían si aplicamos PROCAM.

\section{CONCLUSIONES}

A pesar de que ambos métodos de cálculo de riesgo, tienen unos coeficientes de correlación superiores a 0,75 y que la media de las diferencias es de sólo 2,2\%, existe una gran variabilidad en las diferencias individuales, sobre todo a medida que aumenta el riesgo, como se aprecia en el gráfico de Bland y Altman. Para riesgos bajos, PROCAM infravalora el riesgo, mientras que para riesgos altos lo supravalora comparado con Framingham por Categorías, en una población de varones entre 45-65 años; globalmente los dos métodos son muy parecidos pero a nivel individual no miden lo mismo, lo cual puede tener repercusiones importantes a la hora de tomar decisiones clínico-terapéuticas.

Es necesario realizar estudios nacionales ya que podemos estar supraestimando el riesgo cardiovascular de nuestros pacientes, ya que estos métodos de riesgo están realizados en poblaciones con mayor prevalencia de morbi-mortalidad que la española.

CORRESPONDENCIA:

Artenio Álvarez Cosmea

C/ Rafael Altamira 5, $11^{\circ} \mathrm{A}$

33006 Oviedo

\section{Bibliografía}

1. Anderson KM, Wilson PWF, Odell PM, Kannel WB. An Updated coronary risk profile. A Statement for health profesionals. Circulation 1991; 83: 356-62.

2. Wilson Peter WF, Dàgostino R, Levy D, Belanger A, Silbershatz H, Kannel W. Prediction of Coronary Heart Disease Using Risk Factor categories. Circulation 1998; 97: 1837- 47.

3. Grundy S, Pasternak R, Greenland PH, Smith S, Fuster V. Assessment of Cardiovascular Risk by use of MultipleRisk-Factor Assesment Equations. ACC/AHA Scientific Statement. Circulation 1999; 100: 1481-92.

4. Grundy S, Bazaarre T, Cleeman J, D’Agostino R, Hill M, Houston- Miller N, et al. Prevention Conference V: Beyond secondary prevention: identifying the patient for primary prevention: medical office assessment: Writing Group. Circulation 2000; 101: E3-E11.

5. D`Agostino R, Russell H, Huse D, Ellison R, Silbershatz H, Wilson $\mathrm{P}$, et al. Primary and subsequent coronary risk appraisal: New Results from the Framingham Study. Am Heart J 2000, 139. 272-81.

6. Wood D, De Backer G, Faergeman O, Graham I, Mancia G, Pyörala K. Task Force Report . Prevention of coronary heart disease in clinical practice: Recomendations of the second joint task force of the joint European Societies on coronary prevention. Eur Heart J 1998;19: 1434-503.

7. Wood D, Durrington P, Poulter N, Mcinnes G, Rees A, Wray R. Joint British recomendations on prevention of coronary heart disease in clinical practice. Heart 1998; 80 Suppl 2: 1-29.

8. British Cardiac Society, British Hyperlipidaemia Association, British Hypertension Society, British Diabetic Asso- ciation. Joint British recommendations on prevention of coronary heart disease in clinical practice: Summary. BMJ 2000; 320: 705-8.

9. Jackson R. Updated New Zaeland cardiovascular disease risk- benefit prediction guide. BMJ, 2000; 320: 709-10.

10. Wallis EJ, Ramsay LE, Haq IU, Ghahramani P, Jackson PR, Rowland-Yeo K, et al. Coronary and cardiovascular risk estimation for primary prevention: validation of a new Sheffield table in the 1995 Scottish health survey population. BMJ 2000; 320: 671-6.

11. The International Task Force for Prevention of Coronary Heart Disease. Coronary Heart Disease: Reducing the Risk: the scientific background for primary and secondary prevention of coronary heart disease. Nutr Metab Cardiovasc Dis 1998; 8: 205-71.

12. Assman G, Cullen P, Schulte H. The Münster Heart Study (PROCAM). Results of follow- up at 8 years. Eur Heart J 1998; 19 Suppl A: 2-11.

13. International Task Force for Prevention of Coronary Heart Disease.(www.chd-taskforce.com).

14. V Informe (JNC) Joint National Committee. The fifth report of the Joint National Committee on detection, evaluation, and treatment of high blood pressure (JNC-V). Arch Intern Med 1993; 153: 154-83.

15. Summmary of the second report of the National Cholesterol Education Program (NCEP). Expert Panel on Detection, Evaluation, and Treatment of High Blood Cholesterol in Adults. JAMA 1993; 269: 3015-23.

16. Haq IU, Ramsay LE, Yeo WW, Jackson PR, Wallis EJ. Is the Framingham Risk Funtion valid for Northern European 
populations? A comparasion of methods for estimating absolute coronary risk in high risk men. Heart 1999; 81: 40- 46.

17. Menotti A, Pudd PE, Lanti M. Comparison of the Framingham risk function- based coronary chart with risk function from an Italian population study. European Heart Journal 2000; 21: 365-70.

18. Elosua R, Belmonte E, Sentí M, Masiá R, Marrugat J. ¿Debe diseñarse una estrategia específica de prevención primaria de la cardiopatía isquémica para los países mediterráneos de Europa? Clin Invest Arteriosclerosis 2000; 12: 43-55.

19. D'Agostino RB. Validation of the Framingham Coronary Heart Disease Prediction Scores. Resulst of a Multiple Ethnic Groups Investigation. JAMA 2001; 286: 180-7.

20. Argimón Pallas JM, Jiménez Villa J. Medición de variables.
En: Argimón Pallas JM, Jiménez Villa J, editores. Métodos de investigación aplicados a la atención primaria de salud. Barcelona: Doyma, 1991. p. 109-21.

21. Ripollés Orti M, Martín Rioboo E, Díaz Moreno A, Aranguren Baena B, Murcia Simón M, Toledano Medina A, et al. Concordancia en la medición de presión arterial entre diferentes profesionales sanitarios. ¿Son fiables los esfigmomanómetros de mercurio? Atención Primaria 2001; 27: 234-43.

22. Bland JM, Altman DG. Statical methods for assessing agreement between two methods of clinical measurement. Lancet 1986; 1: 307-10.

23. Latour J, Abraira V, Cabello J, López Sánchez J. Las mediciones clínicas en cardiología: validez y errores de medición. Rev Esp Cardiol 1997; 50: 117-28. 